\title{
Recommendation in Academia: A Joint Multi-Relational Model
}

\author{
Zaihan Yang Dawei Yin Brian D. Davison \\ Department of Computer Science and Engineering, Lehigh University \\ Bethlehem, PA 18015 \\ Email: \{zay206, day207, davison\}@cse.lehigh.edu
}

\begin{abstract}
In this paper, we target at four specific recommendation tasks in the academic environment: the recommendation for author coauthorships, paper citation recommendation for authors, paper citation recommendation for papers, and publishing venue recommendation for author-paper pairs. Different from previous work which tackles each of these tasks separately while neglecting their mutual effect and connection, we propose a joint multi-relational model that can exploit the latent correlation between relations and solve several tasks in a unified way. Moreover, for better ranking purpose, we extend the work maximizing MAP over one single tensor, and make it applicable to maximize MAP over multiple matrices and tensors. Experiments conducted over two real world data sets demonstrate the effectiveness of our model: 1) improved performance can be achieved with joint modeling over multiple relations; 2) our model can outperform three state-of-the art algorithms for several tasks.
\end{abstract}

Keywords: Recommender systems, matrix/tensor factorization, joint modeling, MAP, latent factor model

\section{INTRODUCTION}

People can conduct many activities in academic environment: publishing papers, collaborating with other authors, or citing other papers/authors. Theses activities are sometimes not easy to fulfill. For example, reading and therefore citing new published papers is one of the most important tasks that a researcher should conduct for research, however, to find relevant and referential scientific literature from hundreds of thousands of publications is a time-consuming and laborintensive task especially with the rapid development of Internet which makes published papers easy to be accessed. To better facilitate such activities, information needs have arisen for developing systems that can automatically help to predict or recommend proper venues to submit, papers to cite, and authors to collaborate. In this paper, we focus on the prediction task in academic environment, and particularly pay attention to the following four tasks: the prediction on publishing venues, collaborators, cited papers for authors, and cited papers for papers.

Even though individual systems or algorithms have been proposed to tackle each of the four tasks separately, which we will review in later sections, limitations still remain. Most of the previous methods only focus on one single type of relationship while neglect to explore the mutual interaction among different relationships. In a real complicated academic environment, which often consists of heterogeneous nodes and links, each scientific factor can play different roles, and participate in different activities.

To better model this multi-relational academic activities and to provide good recommendations, several challenges remain: (1) coupled high order data: The entire academic network is composed of multiple relations that mutually affect each other. It is quite natural for a paper that has a large number of citations from other papers to be cited by more authors. (2) cold start problem: the cold start problem is a typical problem in recommender systems. Take the task of citation recommendation for papers as one example, some most recently published papers will hardly be cited since they have never been cited before by other papers or authors, even though they are highly relevant to a topic or may have great contribution in a certain field. (3) Personalization support for authors: Researchers play an important role in many activities, and they may have different preferences in selecting which paper to cite, or which venue to submit, even though those papers or venues focus on similar topics. (4) Interest evolution for authors: The interest of researchers evolves over time. Even though they keep on working in one research field, their research focus and methods may change.

To tackle these challenges (refer to our report [23] for more discussion on this), we propose a joint multi-relational model referred as the JMRM model which directly models several groups of coupled activities in the academic environment and provide a more general framework that can solve several prediction tasks simultaneously in a unified way.

Our model is fundamentally the latent factor collaborativefiltering(CF)-based model, in which each relation can be represented as a matrix or higher-dimensional matrix. However, the following three characteristics distinguish our model from previous ones. Firstly, our model is composed of multiple matrices or tensors, each of which indicate one relation in the academic environment, and are highly coupled with each other. Secondly, we integrate the temporal information into the generation of several matrices to better reflect the involution over authors' preferences; Thirdly, we choose the objective function for solving the model as maximizing the mean average precision (MAP) as compared to most of the previous work minimizing the predicting error (RMSE). MAP is a ranking-based standard IR measure for which errors at top of the ranking list will lead to a higher penalty than errors at lower places of the ranking list. This top-heavy biased property makes MAP particularly suitable to work as the objective function for recommender systems, since most people will only pay attention to the top ranked results in the recommendation list. For this reason, we choose to maximize MAP as our objective function.

To sum up, the main contribution of our work are as follows: (1) we propose a joint multi-relational model which integrates several coupled relations in an academic environment. (2) we choose to maximize MAP as the objective function for solving the model, and extend the tensor factorization approach optimizing MAP into a more general framework that can maximize MAP for coupled multiple matrices and tensors. (3) experimental evaluation over two real world data sets demonstrate 
the capability of our model in four recommendation tasks, as they show improved performance as compared to three stateof-the-art algorithms.

\section{RELATED WORK}

Recently, researchers have explored to enhance the traditional latent factor models by incorporating additional features or content of participating entities. Typical works include the 'Regression Based Factor Models' [1], the CTR model [20], and the 'feature-based matrix factorization' [3] model. However, all of these three models can only cope with the two-order data interactions, and cannot be model higher-order data structures.

The second direction of development for latent factor model emphasizes on joint modeling multi-relational relations. One typical work: the 'collective matrix factorization' model [17] however is only limited to be two or three relations. Most recently, Yin et al. [24] proposed a 'Bayesian probabilistic relational-data Analysis' (BPRA) model which extends the BPMF and BPTF model by making it applicable to arbitrary order of coupled multi-relational data structures. However, the model is based upon point-wise RMSE optimization, different from our targeted ranking-based optimization.

Several ranking-based optimization models have been proposed to replace optimizing point-wise measures, such as RMSE or MSE. One typical work: the 'Bayesian Personalized Ranking' (BPR) model [13] minimizes the AUC metric by using a smooth version of the hinge loss. The method that is most similar to our work is the TFMAP model [15], which proposes a method to approximate and optimize the MAP measure. However, their model is for user-item-context recommendation, and is only able to deal with one single tensor relation, which are both different from our work in this paper.

We then summarize some relevant work with each specific recommendation task considered in this paper. Future paper citation recommendation is the most widely explored problem. We categorized existing works into three groups. In the first group, neighborhood based CF models along with graphbased link prediction approaches are widely used to tackle the citation recommendations for a given author or paper with a partial list of initial citations provided, typical works in this category include [11], [26], [18] and more. In the second group of approach, probabilistic topic modeling is used for citation list generation. In the third group, citation context (the text around citation mentions) is utilized. Typical work includes the context-award citation recommendation work and its extensions proposed by $\mathrm{He}$ et al. [7] and [6]. Despite of these existing work, few work has be developed using CF latent factor models for recommendation, excluding the CTR model. Coauthor-ship recommendation is mostly tackled by using graph-based link prediction approach. The most representative work is proposed by Liben-Nowell [10], which measures the performance on using several graph-based metrics. The work on predicting future conference(venue) submission is seldom explored. Yang et al. [21] proposed an extended version of the neighborhood CF model to solve this problem recently by incorporating stylometric features of papers and distinguishing the importance of four different types of neighboring papers. Similar to the paper citation work, few existing work applies the latent factor model based CF approach, which is different from the work in this paper.
TABLE I

NOTATIONS

\begin{tabular}{|ll|}
\hline$K$ & Number of entity types $(K=6)$ \\
& represents author, citing paper \\
& cited paper, venue, word \\
$a_{i}$ & and feature entity type respectively. \\
$k$ & entity of type $a$ with index $i$ \\
$N_{k}$ & entity type. $k \in a, p, p_{c}, v, w, a_{f}$ \\
$D$ & Number of entities of type $k$ in data corpus \\
$V$ & Dimension for latent vector \\
$\theta_{k}$ & Number of relations $(V=6)$ \\
$\theta_{k_{t}}$ & Latent matrix for entities of type $k$ \\
\hline
\end{tabular}

III. Joint Multi-Relational Model (JMRM): Model DESIGN AND GENERATION

The model is designed for four particular recommendation tasks in the academic environment, each of which represents one academic activity, and induces one relation. Therefore, we have four main relations in the model: the author-papervenue relation (represented as the APV-tensor), author-authorcollaboration relation (AA-matrix), author-paper-citation relation (AP-matrix), and paper-paper-citation relation (PPmatrix). Figure 1 shows the framework of the model. In order to deal with the cold start problem and better support authors' personalization, we further incorporate additional features for papers and authors. In the current work, we only consider the pure paper content as paper features, and we use the PWmatrix to represent it. We model authors and their features as the AF-matrix, and will introduce more detailed features for authors in the following section.

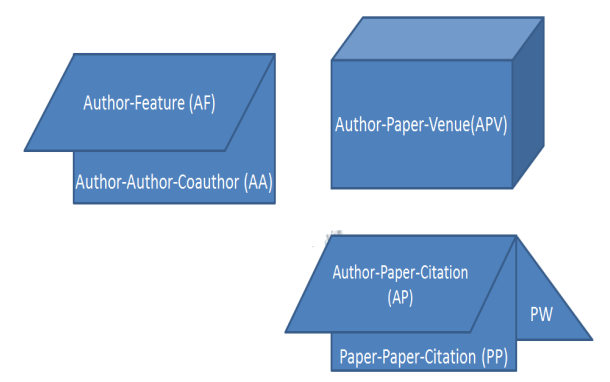

Fig. 1. Coupled Matrices and Tensor

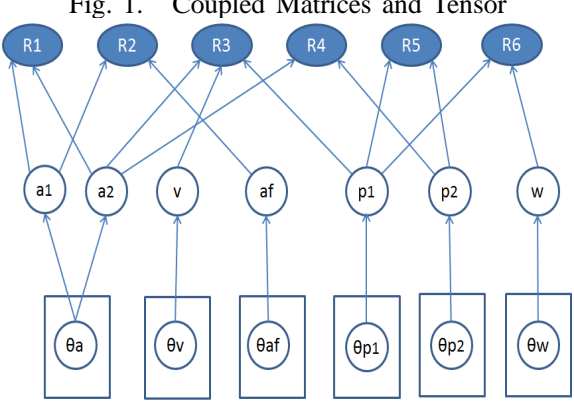

Fig. 2. Graphical Model Representation

We formally describe as follows the four recommendation/prediction tasks and introduce how the corresponding relation is constructed.

Task 1 Publishing venue prediction: this task involves predicting the publishing venue $v_{k}$ for a given author-paper pair $\left(a_{i}, p_{j}\right)$ such that paper $p_{j}$ written by author $a_{i}$ is published in venue $v_{k}$. 
Corresponding relation APV-tensor: the author-papervenue relation This three-order relation consisting of triples <author-paper-venue> indicates the publishing venue selection for papers with known authors.we have: $A P V\left(a_{i}, p_{j}, v_{k}\right)=1$ if paper $p_{j}$ written by author $a_{i}$ is published in venue $v_{k}$; Otherwise, $A P V\left(a_{i}, p_{j}, v_{k}\right)=0$.

Task 2 Recommend paper citations for authors: this task involves recommend a set of papers $\boldsymbol{p}$ for a given author $a_{i}$ such that each paper $p_{j}\left(p_{j} \in \boldsymbol{p}\right)$ is cited at least once by author $a_{i}$.

Corresponding relation AP-matrix: the author-papercitation relation The AP matrix models the citation relationship between authors and papers. An author may cite a paper multiple times at different times. In order to model this temporal evolution property, we first generate a three-order tensor incorporating the temporal factor as the third dimension, and then collapse the tensor into a two-dimensional matrix by aggregating the number of citations at different years with a time decay function. Given an author $a_{i}$, and a paper $p_{j}$ cited by $a_{i}$, the number of times $p_{j}$ is cited by $a_{i}$ on year $t_{k}$ can be retrieved as: $E\left(a_{i}, p_{j}, t_{k}\right)=\sum_{p_{a i} \in p_{a i}} \delta\left(y\left(p_{a i}\right)=\right.$ $\left.t_{k} \wedge p_{j} \in \boldsymbol{c}_{\boldsymbol{p}_{a i}}\right)$ where $p_{a i}$ is any paper published by $a_{i}, \boldsymbol{p}_{a i}$ is the publication set of $a_{i} . c_{p_{a i}}$ is the set of all cited papers of $p_{a i}$. Function $y\left(p_{a i}\right)$ retrieves the publication year of $p_{a i}$, and $\delta(c)$ is a function which returns 1 if condition $c$ is satisfied and 0 otherwise. We aggregate the citations at different time points based upon the hypothesis that authors' interests decay over time, and therefore more recent citation contribute more heavily than older citation. We penalize the old citations by introducing an exponential decay kernel function. The entry $\left\langle a_{i}, p_{j}\right\rangle$ for the collapsed author-paper matrix can thus be defined as: $E_{A P}\left(a_{i}, p_{j}\right)=\sum_{t_{k}=T_{1}}^{T_{2}} e^{-\beta\left(T_{2}-t_{k}\right)} \cdot E\left(a_{i}, p_{j}, t_{k}\right)$ where $T_{1}$ and $T_{2}$ are the earliest and last year for paper publication in the data set, and $\beta$ is the decay rate.

Task 3 Recommend coauthors for authors: this task involves recommend a set of authors $\boldsymbol{a}$ for a given author $a_{i}$ such that for each author $a_{j} \in \boldsymbol{a}$, there exists a coauthorship between $a_{i}$ and $a_{j}$.

Corresponding relation AA-matrix: the author-authorcollaboration relation The AA-matrix indicates the collaboration, an important social interactions between pairs of authors. Similar to the changing interests' of authors over papers, researchers may also change to work with others researchers in different time periods. We follow the same procedure as introduced for the AP-matrix generation by first constructing the author-author-time tensor, and then collapse it into authorauthor matrix. The entry for $\left\langle a_{i}, a_{j}\right\rangle$ can thus be determined by: $E_{A A}\left(a_{i}, a_{j}\right)=\sum_{t_{k}=T_{1}}^{T_{2}} e^{-\beta\left(T_{2}-t_{k}\right)} \cdot E\left(a_{i}, a_{j}, t_{k}\right)$ where $E\left(a_{i}, a_{j}, t_{k}\right)$ is the number of times author $a_{i}$ collaborates with $a_{j}$ on year $t_{k}$.

Task 4 Recommend paper citations for papers: this task involves recommend a set of papers $\boldsymbol{p}$ for a given paper $p_{i}$ such that each paper $p_{j}\left(p_{j} \in \boldsymbol{p}\right)$ is cited at least once by paper $p_{i}$.

Corresponding relation PP-matrix: the paper-paper-citation relation The generation of the PP-matrix is different from that of the AP-matrix or AA-matrix, since each paper can only cite another paper once. However, there also exists temporal influence, as a paper may cite a paper published long time ago, or a more recent one. Suppose we have three papers $p 1, p 2$ and $p 3$, published in $y 1, y 2$ and $y 3$ respectively $(y 1 \leq y 2 \leq y 3)$, and we have paper $p 3$ cites $p 2$ and $p 1$. In our work, we assume that $p 2$ will have a greater contribution in presenting the topic interests or preferences for $p 3$ than $p 1$, since in publishing papers, we often need to review and compare with those most recently published and state-of-theart papers. With this assumption, we have for each entry $<p_{i}, p_{j}>$ indicating that paper $p_{i}$ cites $p_{j}$ in the PP-matrix as: $E_{P P}\left(p_{i}, p_{j}\right)=e^{-\beta\left(y\left(p_{i}\right)-y\left(p_{j}\right)\right)}$, where $y\left(p_{i}\right)$ and $y\left(p_{j}\right)$ returns the publishing year for $p_{i}$ and $p_{j}$ respectively.

PW-matrix: the paper-word relation $\mathrm{PW}$-matrix indicates the features of papers. In current work, we only consider the pure content of papers, and therefore we collect the top returned words in the data set with higher frequency. Each entry of $\left\langle p_{i}, w_{j}\right\rangle$ indicates the term frequency of word $w_{j}$ in paper $p_{i}$.

AF-matrix: the author-feature relation We identify 20 distinctive features for authors listed in Table II to represent the personalized property of an author from three aspects.

TABLE II

AUTHOR FEATURES

\begin{tabular}{|l|l|}
\hline Feature Category & Feature \\
\hline Simple bibliographic & total publicationNo ; total citationNo ; H-index [8]; \\
& G-index [4]; Rational H-index distance [14]; \\
& Rational H-index X [14]; E-index [25]; \\
& Individual H-index [2]; Normalized individual H-index [5] \\
\hline Network-based & $\begin{array}{l}\text { PageRank score on coauthor network; } \\
\text { PageRank score on citation network }\end{array}$ \\
\hline Temporal-based & CareerTime [22]; LastRestTime [22]; \\
& PubInterval [22]; Citation Influence Ratio [22]; \\
& Contemporary H-index [16]; AR-index [9] \\
& AWCR-index [5]; Avg Publication number; \\
& avg Citation number \\
\hline
\end{tabular}

\section{Joint Multi-Relational Model: Algorithm}

\section{A. Preliminary Notations}

As shown in Figure 1, our joint multi-relational model consists of six relations generated by authors, papers, venues, words and features entities. It is noticeable to mention that we distinguish the 'paper' entity into two different entities types: the citing papers and cited papers, and therefore we altogether have six entity types.

In the joint multi-relational model, each entity in the interactions can be represented by a latent feature vector in $\mathbb{R}^{D}$, where $D$ is typically a small number. By doing this, each tensor or matrix can be factorized into lower rank approximations. Figure 2 shows the graphical model for the data factorization associated with our model. The lower-dimensional latent vectors are denoted by $\boldsymbol{\theta}=\left(\theta_{1}, \ldots, \theta_{K}\right)(K=6)$, where for each $k \in K \theta_{k}=\left(\boldsymbol{\theta}_{k 1}, \ldots, \boldsymbol{\theta}_{k N_{k}}\right) \in \mathbb{R}^{N_{k} \times D}$.

\section{B. Model Factorization maximizing MAP}

1) Computing MAP: We choose to maximize MAP as our objective function due to its top-heavy bias property. Two questions remain for incorporating MAP into matrix/tensor factorization: how to represent the 'rank' of the entities and therefore compute the MAP scores based upon the latent feature vectors. We follow the same idea proposed in paper [15] to smoothly approximate MAP, and make it appropriate to be used for both tensors and matrices. Since our model contains one tensor and five matrices, for better illustration, we 
choose to take the APV-tensor and AP-matrix as two examples to show how to compute the MAP scores. The same method can be applied to the other four matrices.

In a tensor like APV-tensor, the real value for entry $<a_{i}, p_{j}, v_{m}>$ is presented as $f_{A P V_{a_{i} p_{j} v_{m}}}$, the predicted value can be computed as: $\hat{f}_{a_{i} p_{j} v_{m}}=\left\langle\theta_{a_{i}}, \theta_{p_{j}}, \theta_{v_{m}}\right\rangle=$ $\sum_{d=1}^{D} \theta_{a_{i} d} \theta_{p_{j} d} \theta_{v_{m} d}$, where $D$ is the dimension for latent vector.

Similarly, In a matrix like AP-matrix, the real value for entry $<a_{i}, p_{j}>$ is presented as $f_{A P_{a_{i} p_{j}}}$, and the predicted value can be computed as: $\hat{f}_{a_{i} p_{j}}=\left\langle\theta_{a_{i}}, \theta_{p_{j}}\right\rangle=\sum_{d=1}^{D} \theta_{a_{i} d} \theta_{p_{j} d}$.

Under these schemes, suppose $v_{m}$ in triple $<a_{i}, p_{j}, v_{m}>$ is the entity that needs to be ranked, and $p_{j}$ in tuple $<a_{i}, p_{j}>$ is the entity that needs to be ranked, then we can directly approximate $1 / r_{a_{i} p_{j} v_{m}}$ for $v_{m}$ and $1 / r_{a_{i} p_{j}}$ for $p_{j}$ by: $\frac{1}{r_{a_{i} p_{j} v_{m}}} \approx g\left(\hat{f}_{a_{i} p_{j} v_{m}}\right)=g\left(\left\langle\theta_{a_{i}}, \theta_{p_{j}}, \theta_{v_{m}}\right\rangle\right)$ and $\frac{1}{r_{a_{i} p_{j}}} \approx g\left(\hat{f}_{a_{i} p_{j}}\right)=g\left(\left\langle\theta_{a_{i}}, \theta_{p_{j}}\right\rangle\right)$, where function $g(\cdot)$ is the sigmoid function satisfying $g(x)=\frac{1}{1+e^{-x}}$.

Correspondingly, the loss function in terms of the MAP values for APV-tensor and AP-matrix can be computed as in Equation 1:

To compute the loss function for matrix AA, PP, PW and $\mathrm{AF}$, we can follow the same way as we do for the AP matrix.

$$
\begin{aligned}
L_{a p v} & =M A P_{a p v}=\frac{1}{N_{a} N_{p}} \sum_{i=1}^{N_{a}} \sum_{j=1}^{N_{p}} \frac{1}{\sum_{t=1}^{N_{v}} f_{A P V_{a_{i} p_{j} v_{m}}}} \\
& \times \sum_{t 1=1}^{N_{v}} f_{A P V_{a_{i} p_{j} v_{t 1}}} g\left(\left\langle\theta_{a_{i}}, \theta_{p_{j}}, \theta_{v_{t 1}}\right\rangle\right) \\
& \times \sum_{t 2=1}^{N_{v}} f_{A P V_{a_{i} p_{j} v_{k 2}}} g\left(\left\langle\theta_{a_{i}},\left(\theta_{v_{t 2}}-\theta_{v_{t 1}}\right), \theta_{p_{j}}\right\rangle\right) \\
& =M A P_{a p}=\frac{1}{N_{a}} \sum_{i=1}^{N_{a}} \frac{1}{\sum_{j=1}^{N_{p}} f_{A P_{a_{i} p_{j}}}} \\
& \times \sum_{t 1=1}^{N_{p}} f_{A P_{a_{i} p_{t 1}}} g\left(\left\langle\theta_{a_{i}}, \theta_{p_{t 1}}\right\rangle\right) \\
& \times \sum_{t 2=1}^{N_{p}} f_{A P_{a_{i} p_{t 2}}} g\left(\left\langle\theta_{a_{i}},\left(\theta_{P_{t 2}}-\theta_{P_{t 1}}\right)\right\rangle\right)
\end{aligned}
$$

2) Optimization: The overall loss function for this multirelational model is the summation over all individual loss functions plus the regularization terms to prevent over-fitting, We use $\Omega$ to denote the regularization terms, where $\|\cdot\|$ indicates the Frobenius norms.

$$
\begin{aligned}
& L=L_{A P V}+L_{A A}+L_{A P}+L_{P P}+L_{P W}+L_{A F}+\Omega \\
& \Omega=\sum_{k \in a, p, p_{c}, v, w, a_{f}} \frac{\lambda_{\boldsymbol{\theta}_{\boldsymbol{k}}}}{2}\left\|\boldsymbol{\theta}_{\boldsymbol{k}}\right\|^{2}
\end{aligned}
$$

We choose to use gradient ascent to solve this optimization problem. To jointly model multiple relations, the gradients for the same entity across different relations will be merged. For better illustration, we list below the gradients for the author, paper and venue entity in the APV-tensor, and author and paper entity in the AP-matrix. Similar process can be applied into other entities in other relations.

For one particular author $a_{i}$, paper $p_{j}$ and venue $v_{m}$ in the APV-tensor, the gradients for updating their corresponding latent vector $\theta_{a_{i}}, \theta_{p_{j}}$ and $\theta_{v_{m}}$ can be computed as follows. For notation convenience, we adopt the following substitutions:

$$
\begin{aligned}
& \hat{f}_{A P V_{a_{i} p_{j} v_{m}}}=\left\langle\theta_{a_{i}}, \theta_{p_{j}}, \theta_{v_{m}}\right\rangle \hat{f}_{A P V_{a_{i} p_{j}}\left(v_{t_{2}}-v_{t_{1}}\right)}= \\
& \left\langle\theta_{a_{i}}, \theta_{p_{j}},\left(\theta_{v_{t_{1}}}-\theta_{v_{t_{1}}}\right)\right\rangle \\
& \frac{\partial L_{A P V}}{\partial \theta_{a_{i}}}=\sum_{s=1}^{N_{p}} \frac{1}{\sum_{t=1}^{N_{v}} f_{A P V_{a_{i} p_{s} v_{t}}}} \sum_{t_{1}=1}^{N_{v}} f_{A P V_{a_{i} p_{s} v_{t_{1}}}} \\
& \times \quad\left[\delta_{1}\left(\theta_{p_{s}} \odot \theta_{v_{t_{1}}}\right)+g\left(\hat{f}_{A P V_{a_{i} p_{s} v_{t_{1}}}}\right)\right. \\
& \times \sum_{t_{2}=1}^{N_{v}} f_{A P V_{a_{i} p_{s} v_{t_{2}}}} g^{\prime}\left(\hat{f}_{\left.A P V_{a_{i} p_{s}\left(v_{t_{2}}-v_{t_{1}}\right)}\right)}\right) \\
& \left.\times \quad\left(\theta_{p_{s}} \odot \theta_{v_{t_{2}}}\right)\right]-\lambda \theta_{a_{i}} \\
& \frac{\partial L_{A P V}}{\partial \theta_{p_{j}}}=\sum_{s=1}^{N_{a}} \frac{1}{\sum_{t=1}^{N_{v}} f_{A P V_{a_{s} p_{j} v_{t}}}} \sum_{t_{1}=1}^{N_{v}} f_{A P V_{a_{s} p_{j} t_{1}}} \\
& \times \quad\left[\delta_{1}\left(\theta_{a_{s}} \odot \theta_{v_{t_{1}}}\right)+g\left(\hat{f}_{A P V_{a_{s} p_{j} v_{t_{1}}}}\right)\right. \\
& \times \sum_{t_{2}=1}^{N_{v}} f_{A P V_{a_{s} p_{j} v_{t_{2}}}} g^{\prime}\left(\hat{f}_{\left.A P V_{a_{s} p_{j}\left(v_{t_{2}}-v_{t_{1}}\right)}\right)}\right) \\
& \left.\times \quad\left(\theta_{a_{s}} \odot \theta_{v_{t_{2}}}\right)\right]-\lambda \theta_{p_{j}} \\
& \frac{\partial L_{A P V}}{\partial \theta_{v_{m}}}=\sum_{s=1}^{N_{a}} \sum_{d=1}^{N_{p}} \frac{f_{A P V_{a_{s} p_{d} v_{m}}}\left(\theta_{a_{s}} \odot \theta_{p_{d}}\right)}{\sum_{t_{1}=1}^{N_{v}} f_{A P V_{a_{s} p_{d} v_{1}}}} \\
& \times \sum_{t_{2}=1}^{N_{v}} f_{A P V_{a_{s} p_{d} t_{2}}}\left[g^{\prime}\left(\hat{f}_{A P V_{a_{s} p_{d} v_{m}}}\right)\right.
\end{aligned}
$$

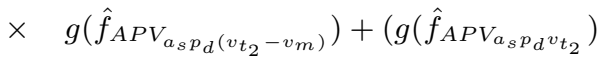

$$
\begin{aligned}
& \text { - } \left.g\left(\hat{f}_{A P V_{a_{s} p_{d} v_{m}}}\right)\right) g^{\prime}\left(\hat{f}_{\left.\left.A P V_{a_{s} p_{d}\left(v_{t_{2}}-v_{m}\right)}\right)\right]}\right) \\
& -\lambda \theta_{v_{m}}
\end{aligned}
$$

where

$\delta_{1}=g^{\prime}\left(\hat{f}_{A P V_{a_{i} p_{j} v_{m}}}\right) \sum_{t_{1}=1}^{N_{v}} f_{A P V_{a_{i} p_{j} v_{t_{1}}}} g\left(\hat{f}_{\left.A P V_{a_{i} p_{j}\left(v_{t_{1}}-v_{m}\right)}\right)}\right)-$

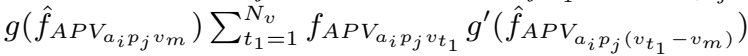

For one author $a_{i}$ and paper $p_{j}$ in the AP-matrix:

$$
\begin{aligned}
\frac{\partial L_{A P}}{\partial \theta_{a_{i}}} & =\frac{1}{\sum_{t=1}^{N_{p}} f_{A P_{a_{i} p_{t}}}} \sum_{t_{1}=1}^{N_{p}} f_{A P_{a_{i} p_{t}}}\left[\delta_{2}\left(\theta_{p_{t_{1}}}\right)\right. \\
& \left.+g\left(\hat{f}_{A P_{a_{i} p_{t_{1}}}}\right) \sum_{t_{2}=1}^{N_{p}} f_{A P_{a_{i} p_{t_{2}}}} g^{\prime}\left(\hat{f}_{\left.A P_{a_{i}\left(p_{t_{2}}-p_{t_{1}}\right)}\right)}\right)\left(\theta_{p_{t_{2}}}\right)\right] \\
& -\lambda \theta_{a_{i}} \\
\frac{\partial L_{A P}}{\partial \theta_{p_{j}}} & =\sum_{s=1}^{N_{a}} \frac{f_{A P_{a_{s} p_{j}}}\left(\theta_{a_{s}}\right)}{\sum_{t_{1}=1}^{N_{p}} f_{A P_{a_{s}} p_{t_{1}}}} \times \sum_{t_{2}=1}^{N_{p}} f_{A P_{a_{s} p_{t_{2}}}}\left[g^{\prime}\left(\hat{f}_{A P_{a_{s} p_{j}}}\right)\right. \\
& \times g\left(\hat{f}_{\left.A P_{a_{s}\left(p_{t_{2}}\right.}-p_{j}\right)}\right)+\left(g\left(\hat{f}_{A P_{a_{s}} p_{t_{2}}}\right)\right. \\
& \left.-g\left(\hat{f}_{A P_{a_{s} p_{j}}}\right)\right) g^{\prime}\left(\hat{f}_{\left.\left.A P_{a_{s}\left(p_{t_{2}}-p_{j}\right)}\right)\right]}\right) \\
& -\lambda \theta_{p_{j}}
\end{aligned}
$$

where $\delta_{2}=g^{\prime}\left(\hat{f}_{A P_{a_{i} p_{j}}}\right) \sum_{t_{1}=1}^{N_{p}} f_{A P_{a_{i} p_{t_{1}}}} g\left(\hat{f}_{\left.A P_{a_{i}\left(p_{t_{1}}-p_{j}\right)}\right)}\right)-$ $g\left(\hat{f}_{A P_{a_{i} p_{j}}}\right) \sum_{t_{1}=1}^{N_{p}} f_{A P_{a_{i} p_{t_{1}}}} g^{\prime}\left(\hat{f}_{\left.A P_{a_{i}\left(p_{t_{1}}-p_{j}\right)}\right)}\right)$

where $g^{\prime}(x)$ is the derivative of $g(x)$ and $\odot$ denotes elementwise product, and $\lambda$ is the regularization parameter.

\section{Recommendation by Factor Matrices}

After retrieving the latent matrix for each entity type, it is straightforward to generate the ranking list based upon the 
TABLE III

Data SeT STATISTICS

\begin{tabular}{lllll}
\hline data set & authors & papers & venues & \\
ACM & 24,764 & 18,121 & 846 & \\
ArnetMiner & 49,298 & 47,794 & 1,682 & \\
\hline data set & APV & AA & AP & PP \\
& record & records & records & records \\
ACM & 47,810 & 112,456 & 366,201 & 71,396 \\
ArnetMiner & 132,186 & 361,794 & $1,675,564$ & 237,531 \\
\hline
\end{tabular}

recommendation task and the design of matrix/tensor. Take the prediction task for the author-paper citation as one example, given one author $a_{i}$, we can achieve the relevance score of each paper $p_{j}$ in the candidate set by computing $\frac{1}{r_{a_{i} p_{j}}} \approx g\left(\hat{f}_{a_{i} p_{j}}\right)=$ $g\left(\left\langle\theta_{a_{i}}, \theta_{p_{j}}\right\rangle\right)$, and rank all papers in descending order. The same process can be applied to all other recommendation tasks considered in our model.

\section{EXPERIMENTAL EVALUATION}

We report in this section the experimental evaluation results for our model, and compare it with several existing state-ofthe-art algorithms.

\section{A. Data Sets}

We conducted experiments over two real world data sets. The ACM data set is a subset of the ACM Digital Library, from which we crawled one descriptive web page for each 172,890 distinct papers having both title and abstract information. For each published paper, we extracted the information about its authors and references. Due to possible author names' ambiguity, we represent each candidate author name by a concatenation of the first name and last name, while removing all the middle names. We then use exact match to merge candidate author names. Finally, we obtain 170,897 distinct authors, and 2097 venues. The ArnetMiner data set is the data set 'DBLP-Citation-network V5' provided by Tsinghua University for their ArnetMiner academic search engine [19]. It is the crawling result from the ArnetMiner search engine on Feb 21st, 2011 and further combined with the citation information from ACM. After carrying out the same data processing method as we did for the ACM data set, we find 1,558,415 papers, 795,385 authors and 6010 venues. For papers in each data set separately, we collect the papers with complete information (authors, abstract, publishing venue and publishing year) and have been cited at least 5 times in the ACM data set and 10 times in the ArnetMiner data set. Based on these papers, we further collect all their authors and publishing venues.

We construct the tensor and matrices as introduced in section 3 for each data set. The $\beta$ parameter in AA, AP and PP matrix is set to be 0.5. The PW-relation and AF-relation are constructed for all valid authors and papers. Table III shows a brief data statistics for both data sets, and the total number of records for each relation. Five-fold cross validation is conducted over the APV-relation, AA-relation, AP-relation and PP-relation to get the averaged predicting results. In the APV-relation, since each paper can have multiple authors but just one publishing venue, in order to avoid to have overlapped records in the training and testing set, we split the APVrelation into five folds by guaranteeing that one particular paper with all its authors (and the associated records) would appear in either the training or the testing set.

We adopted MAP as our evaluation metric, as the model is specially designed for maximizing MAP. Since the data in
TABLE IV

PERFORMANCE COMPARISON OVER DIFFERENT COMBINATIONS OF RELATIONS

\begin{tabular}{|l|llll|}
\hline \multirow{2}{*}{ Combinations } & \multicolumn{4}{|c|}{ ACM } \\
\cline { 2 - 5 } & APV & AA & AP & PP \\
\hline C0 & $\mathbf{0 . 0 3 2 9}^{*}$ & $0.0487^{*}$ & $0.0456^{*}$ & 0.0389 \\
C1 & $0.0263^{*}$ & $\mathbf{0 . 0 5 6 0}$ & $0.0455^{*}$ & $0.0325^{*}$ \\
C2 & $0.0282^{*}$ & $0.0462^{*}$ & $0.0458^{*}$ & $0.0338^{*}$ \\
C3 & $0.0307^{*}$ & $0.0460^{*}$ & $0.0455^{*}$ & $0.0329^{*}$ \\
C4 & $0.0279^{*}$ & NA & NA & NA \\
C5 & NA & $\mathbf{0 . 0 5 6 0}$ & NA & NA \\
C6 & NA & NA & $\mathbf{0 . 0 4 6 5}$ & NA \\
C7 & NA & NA & NA & $\mathbf{0 . 0 3 9 5}$ \\
C8 & NA & $0.0468^{*}$ & $0.0453^{*}$ & $0.0325^{*}$ \\
\hline
\end{tabular}

TABLE V

PERFORMANCE COMPARISON OVER DIFFERENT COMBINATIONS OF RELATIONS

\begin{tabular}{|l|llll|}
\hline \multirow{2}{*}{ Combinations } & \multicolumn{4}{|c|}{ ArnetMiner } \\
\cline { 2 - 5 } & APV & AA & AP & PP \\
\hline C0 & $0.0277^{*}$ & $0.0534^{*}$ & $0.0782^{*}$ & $0.0342^{*}$ \\
C1 & $0.0289^{*}$ & $\mathbf{0 . 0 5 6 6}$ & $\mathbf{0 . 0 7 8 8}$ & $\mathbf{0 . 0 3 5 7}$ \\
C2 & $\mathbf{0 . 0 3 1 7}^{*}$ & $0.0541^{*}$ & 0.0786 & 0.0353 \\
C3 & $0.0285^{*}$ & $0.0538^{*}$ & 0.0784 & 0.0353 \\
C4 & 0.0316 & NA & NA & NA \\
C5 & NA & 0.0565 & NA & NA \\
C6 & NA & NA & 0.0786 & NA \\
C7 & NA & NA & NA & $0.0348^{*}$ \\
C8 & NA & $0.0543^{*}$ & 0.0787 & $0.0349^{*}$ \\
\hline
\end{tabular}

each relation is quite sparse we cannot treat all entries with no observed data as negative samples (consider the situation that paper $a$ should also cite paper $b$, but unfortunately it did not.), in which case the recommendation results would be deteriorated. To avoid this, we randomly select 200 negative samples (much higher than the average node degree in each relation) for each entity in the testing set. The performance is therefore measured based on the recommendation list that contains the known positive samples and 200 randomly selected negative samples.

In all experiments, we set the latent dimensionality $D=10$, the regularization parameter $\lambda=0.001$ and the learning-rate as 0.001 .

\section{B. Co-effects analysis of multiple relations}

In this part of experiments, we work on totally eight different kinds of multi-relational combinations, and evaluate the performance over four tasks respectively. Table IV and V shows the results.

In Table IV and $\mathrm{V}, c_{0}$ indicates the single relation respectively. $c_{1}=\{a p v, a a, a p, p p, p w, a f\}, c_{2}=$ $\{a p v, a a, a p, p p, p w\}, c_{3}=\{a p v, a a, a p, p p\}, c_{4}=$ $\{a p v, p w, a f\}, c_{5}=\{a, a f\}, c_{6}=\{a p, p w, a f\}, c_{7}=$ $\{p p, p w\}$, and $c_{8}=\{a a, a p, p p\}$.

Several observations can be drawn from the results. 1) Under almost all situations, jointly modeling multiple relations can indeed improve the prediction performance. For the four tasks over two data sets (just except the publishing venue prediction (APV) on ACM data set), the best performance is always achieved when some relations are jointly modeled. 2) There is no clear trend that the more relations we jointly modeled, the better performance we can achieve. For some prediction task, i.e., the paper-paper citation prediction on ACM data set, best performance is obtained when only paperpaper-citation and paper-word relation are incorporated. However, for the ArnetMiner data set, three out of four tasks have the best performance with all relations incorporated. 
TABLE VI

PERFORMANCE COMPARISON

\begin{tabular}{|l|llll|}
\hline \multirow{2}{*}{ Approaches } & \multicolumn{4}{|c|}{ ACM } \\
\cline { 2 - 5 } & APV & AA & AP & PP \\
\hline JMRM & $0.0329^{*}$ & $\mathbf{0 . 0 5 6 0}$ & $0.0465^{*}$ & $\mathbf{0 . 0 3 9 5}$ \\
FM & $\mathbf{0 . 2 1 2 7}$ & $0.0434^{*}$ & $0.0388^{*}$ & $0.0053^{*}$ \\
CTR & & $0.0374^{*}$ & $\mathbf{0 . 0 5 1 3}$ & $0.0341^{*}$ \\
BPRA & $0.0161^{*}$ & 0.0558 & $0.0360^{*}$ & $0.0216^{*}$ \\
\hline
\end{tabular}

TABLE VII

PERFORMANCE COMPARISON

\begin{tabular}{|l|llll|}
\hline \multirow{2}{*}{ Approaches } & \multicolumn{4}{|c|}{ ArnetMiner } \\
\cline { 2 - 5 } & APV & AA & AP & PP \\
\hline JMRM & $0.0317^{*}$ & $\mathbf{0 . 0 5 6 6}$ & 0.0788 & $0.0357^{*}$ \\
FM & $\mathbf{0 . 1 5 9 5}$ & $0.0402^{*}$ & $0.0613^{*}$ & $0.0047^{*}$ \\
CTR & & $0.0395^{*}$ & $0.0756^{*}$ & $\mathbf{0 . 0 3 7 5}$ \\
BPRA & $0.0176^{*}$ & $0.0359^{*}$ & $\mathbf{0 . 0 7 9 4}$ & $0.0286^{*}$ \\
\hline
\end{tabular}

For each relation in both of the two data sets, we conducted the students' $t$ test between the best performance result with others. Statistically significant improvements (paired-based $p \leq 0.05)$ are labeled with $\mathrm{a} *$ in Table IV and V.

\section{Comparison with existing methods}

We report the performance comparison with three state-ofthe-art approaches: the Factorization Machines (short as FM) [12], the Collaborative Topic Regression (short as CTR) [20] and the Bayesian probabilistic relational-data Analysis [24] approach.

For FM, CTR and BPRA models, we feed the same training and testing set we used for JMRM, and evaluate the prediction performance on each individual relations separately. For JMRM, the reported results are the best results selected from different combinations of multiple relations (as shown in Table IV). For using FM method, we regard the tasks as 'regression' tasks; The dimensionality of the factorization machine is set to be ' $1,1,8$ ', indicating that the global bias, one-way interactions and pairwise interactions are all used, and that the number of factors used for pairwise interactions is set to be 8. Stochastic gradient descent (SGD) is chosen to used as the learning method. For CTR method, we construct paper profiles by their abstracts, and author profiles by concatenating all their publications. The basic LDA is used to retrieve the topic proportion and distribution vectors. The dimension for latent factor is set to be 10 , and the number of latent topics is set to 20. Since CTR is only proposed for factorizing two types of entities, we did not adopt it to the task of publishing venue prediction (the APV-relation). Note that both FM and CTR are implemented using publicly available software. We also set the dimension for latent factor in BPRA as 10 .

Table VI and VII show the results. As indicated, we found that our JMRM mode can outperform FM and CTR in several cases which demonstrates the effectiveness of our model. FM can achieve significantly better results than JMRM in predicting publishing venue, but has a very poor performance in predicting paper-paper citation. Our model shows the best overall performance, since out of 8 cases (four recommendation tasks over two data sets), our model ranks first for three cases, and the second for the other five cases, demonstrating its superiority in providing recommendations for four tasks simultaneously.

\section{Conclusions}

We proposed an extended latent factor model that can jointly model several relations in an academic environment. The model is specially designed for our recommendation tasks, and is proposed based upon the assumption that several academic activities are highly coupled, and that by joint modeling, we can not only solve the cold start problem but also help in achieving more coherent and accurate latent feature vectors. Moreover, to facilitate ranking, we extend an existing work which directly maximizes MAP over one single tensor into a more generalize form and is therefore able to maximize MAP over several matrices and tensors. Experiments carried out over two real world data sets demonstrate the effectiveness of our model.

\section{REFERENCES}

[1] D. Agarwal and B.-C. Chen. Regression-based latent factor models. In KDD, 2009.

[2] P. Batista, M. Campiteli, and O. Kinouchi. Is it possible to compare researchers with different scientific interests? Scientometrics, 68:179189, 2006.

[3] T. Chen, Z. Zheng, Q. Lu, W. Zhang, and Y. Yu. Feature-based matrix factorization. CoRR, abs/1109.2271, 2011.

[4] Egghe. Theory and practice of the g-index. Scientometrics, 69:131-152, 2006.

[5] A. Harzing. The publish or perish book. 2010.

[6] Q. He, D. Kifer, J. Pei, P. Mitra, and C. L. Giles. Citation recommendation without author supervision. In WSDM, pages 755-764, 2011.

[7] Q. He, J. Pei, D. Kifer, P. Mitra, and L. Giles. Context-aware citation recommendation. In $W W W$, pages $421-430,2010$.

[8] J. Hirsch. An index to quantify an individual's scientific research output. Proc.Nat.Acad.Sci., 46:16569, 2005.

[9] B. Jin. The ar-index: Complementing the h-index. Intl. Socienty for Scientometrics and Informetrics Newsletter, 2007.

[10] D. Liben-Nowell and J. Kleinberg. The link-prediction problem for social networks. J. Am. Soc. Inf. Sci. Technol., 58(7), 2007.

[11] S. M. McNee, I. Albert, D. Cosley, P. Gopalkrishnan, S. K. Lam, A. M. Rashid, J. A. Konstan, and J. Riedl. On the recommending of citations for research papers. In CSCW, 2002.

[12] S. Rendle. Factorization machines with libfm. ACM Trans. Intell. Syst. Technol., 3(3):57:1-57:22.

[13] S. Rendle, C. Freudenthaler, Z. Gantner, and L. Schmidt-Thieme. Bpr: Bayesian personalized ranking from implicit feedback. In UAI, UAI '09, pages 452-461, 2009.

[14] F. Ruane and R. Tol. Rational (successive) h-indices: An application to economics in the republic of iremand. Scienctometrics, 2008.

[15] Y. Shi, A. Karatzoglou, L. Baltrunas, M. Larson, A. Hanjalic, and N. Oliver. Tfmap: optimizing map for top-n context-aware recommendation. In SIGIR, pages 155-164, 2012.

[16] A. Sidiropoulos, D. Katsaros, and Y. Manolopoulos. Generalized hirsch h-index for disclosing latent facts in citation networks. Scientometrics, 72:253-280, 2007.

[17] A. P. Singh and G. J. Gordon. Relational learning via collective matrix factorization. In $K D D, 2008$.

[18] T. Strohman, W. B. Croft, and D. Jensen. Recommending citations for academic papers. In SIGIR, 2007.

[19] J. Tang, J. Zhang, L. Yao, J. Li, L. Zhang, and Z. Su. ArnetMiner: extraction and mining of academic social network. In $K D D, 2008$.

[20] C. Wang and D. M. Blei. Collaborative topic modeling for recommending scientific articles. In $K D D, 2011$.

[21] Z. Yang and B. D. Davison. Venue recommendation: Submitting your paper with style. In ICMLA (1), pages 681-686, 2012.

[22] Z. Yang, D. Yin, and B. D. Davison. Award prediction with temporal citation network analysis. In SIGIR, SIGIR '11, pages 1203-1204, 2011.

[23] Z. Yang, D. Yin, and B. D. Davison. Optimizing map in joint multirelational models for recommendations in academic network. Technical Report Technical Report LU-CSE-14-002, Dept. of Compute Science and Engineering, Lehigh University, 2014.

[24] D. Yin, S. Guo, B. Chidlovskii, B. D. Davison, C. Archambeau, and G. Bouchard. Connecting comments and tags: improved modeling of social tagging systems. In WSDM, pages 547-556, 2013.

[25] C. Zhang. The e-index, complementing the h-index for excess citations. PLos One, 4(5):1-4, 2009.

[26] D. Zhou, S. Zhu, K. Yu, X. Song, B. L. Tseng, H. Zha, and C. L. Giles. Learning multiple graphs for document recommendations. In $W W W$, pages 141-150, 2008. 